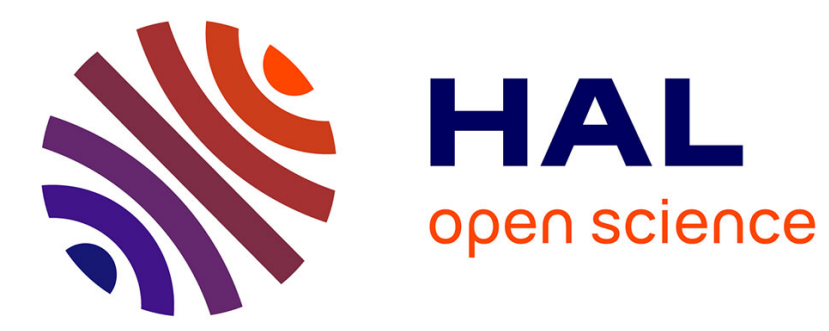

\title{
Performances of some reduced bases for the stability analysis of a disc/pads system in sliding contact
} Denis Brizard, Olivier Chiello, Jean-Jacques Sinou, Xavier Lorang

\section{To cite this version:}

Denis Brizard, Olivier Chiello, Jean-Jacques Sinou, Xavier Lorang. Performances of some reduced bases for the stability analysis of a disc/pads system in sliding contact. Journal of Sound and Vibration, 2011, 330, pp.703-720. 10.1016/j.jsv.2010.09.003 . hal-00625107

\section{HAL Id: hal-00625107 https://hal.science/hal-00625107}

Submitted on 26 Sep 2012

HAL is a multi-disciplinary open access archive for the deposit and dissemination of scientific research documents, whether they are published or not. The documents may come from teaching and research institutions in France or abroad, or from public or private research centers.
L'archive ouverte pluridisciplinaire HAL, est destinée au dépôt et à la diffusion de documents scientifiques de niveau recherche, publiés ou non, émanant des établissements d'enseignement et de recherche français ou étrangers, des laboratoires publics ou privés. 


\title{
Performances of some reduced bases for the stability analysis of a disc/pads system in sliding contact
}

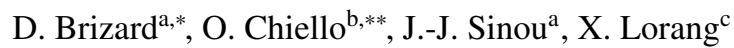 \\ ${ }^{a}$ Laboratoire de Tribologie et Dynamique des Systèmes, UMR-CNRS 5513, Ecole Centrale de Lyon, 36 Avenue Guy de Collongue, 69134 Ecully \\ Cedex, France \\ ${ }^{b}$ Laboratoire Transports et Environnement, Institut National de Recherche sur les Transports et leur Securité, 25 avenue Franois Mitterrand, \\ 69675 Bron Cedex, France \\ ${ }^{c}$ SNCF, Innovative and Reseach Department, Physics of Railway System and Passanger Comfort, 45 rue de Londres, 75379 Paris Cedex 08, \\ France
}

\begin{abstract}
The complex eigenvalue analysis is a widely used technique to investigate the stability of a dynamical system with frictional contact. In the case of brake systems, it is the most frequently employed method to study the propensity of the brake to generate squeal noise. When finite elements models are considered, iterative solvers are needed to calculate the complex modes and eigenvalues with good precision. In practice, reduced real bases are often used in order to reduce the computational times. However, great attention should be focused on the errors introduced by the reduction, which is rarely done. In this paper, the performances of some reduced bases are investigated in the case of a simple disc/pads system. Bases composed of real coupled modes and bases provided by Component Mode Synthesis (CMS) techniques are tested. An enrichment of these bases is proposed in order to improve the precision of the results. In particular, new rubbing attachments modes are proposed to adapt free-interface CMS techniques to frictional contact. When real coupled modes are used, it is suggested to complete the basis by the static response to a first-order approximation of the friction forces. Applied to the disc/pads model, the different enrichment options allow a reduction of the errors on frequencies, divergence rates and mode shapes by a factor comprised between 10 and 100 without significantly increasing the computational times.
\end{abstract}

Keywords:

\section{Nomenclature}

\footnotetext{
${ }^{*}$ Corresponding author

*** Principal corresponding author

Email addresses: denis.brizard@ec-lyon.fr (D. Brizard), olivier.chiello@inrets.fr (O. Chiello)
} 


\begin{tabular}{|c|c|}
\hline$\underline{n}, \underline{t}, \underline{b}$ & normal, tangential and radial directions at a particular contact point \\
\hline$\overline{\mathbf{P}}_{n}, \overline{\mathbf{P}}_{t}$ & projection matrices on the relative displacements between disc and pads along the normal and tangential directions \\
\hline $\mathbf{r}_{n}, \mathbf{r}_{t}$ & normal and tangential reaction forces at the contact nodes \\
\hline $\mathbf{T}_{c}$ & basis for the kernel of the normal contact constraints $\mathbf{P}_{n}$ \\
\hline $\mathbf{T}_{r}$ & some reduced basis or other \\
\hline $\mathbf{M}, \mathbf{K}$ & mass and stiffness matrix of the uncoupled system \\
\hline$\tilde{\mathbf{M}}_{c}, \tilde{\mathbf{K}}_{c}$ & mass and stiffness matrices of the Coupled system with friction \\
\hline $\mathbf{M}_{\mu}, \mathbf{K}_{\mu}$ & mass and stiffness matrices taking into account the effects of the friction forces \\
\hline $\mathbf{C}_{b}$ & radial dissipation effect damping matrix \\
\hline $\mathbf{K}_{c}$ & stiffness matrix of the frictionless coupled system \\
\hline$\mu$ & friction coefficient \\
\hline$\omega_{i}, R_{i}, \xi_{i}$ & imaginary part (frequency), real part and divergence rate associated with mode $i$ \\
\hline$\hat{\omega}_{i}, \hat{\xi}_{i}$ & circular frequency and divergence rate of mode $i$ of reference solution \\
\hline$\Phi, \Psi$ & matrix of normal modes, matrix of static modes \\
\hline$n_{m}, n_{c}$ & number of retained normal Modes, number of Contact nodes \\
\hline Superscripts & \\
\hline$*, \mathrm{~T}$ & conjugate and transpose of a matrix or a vector \\
\hline & related to reference solution \\
\hline$D, P$ & related to Disc and Pins \\
\hline Subscripts & \\
\hline$f, s$ & related to free boundary normal modes and sliding boundary normal modes \\
\hline an, $c n$ & related to Normal Attachment modes and Normal Constrained modes \\
\hline$a t, a \mu$ & related to Tangential Attachment modes and rubbing Attachment modes \\
\hline$R$ & related to the normal modes of the frictionless coupled structure \\
\hline cat & related to the Tangential Attachment modes of the Coupled structure \\
\hline
\end{tabular}

\section{Introduction}

The noise generated by vehicles brakes is one of the most difficult problems with brake systems. Research for predicting and removing brake noise has been regularly performed for many years, especially for the automotive industry $[1,2,3,4,5]$. Among the different encountered brake noises, the squeal noise is characterized by very annoying spectra with one or several pure frequencies in the medium or high frequency range $(1-15 \mathrm{kHz})$. Despite great progress in the modeling of brake squeal, many of the noise abatement approaches are still individual and empirical. It is therefore an important issue to be able to predict this noise.

Friction between two structures in contact can generate many kinds of vibrations and noise [6]. Some of them are forced vibrations, for instance due to the macroscopic roughness of the surfaces in contact. Others are parametric vibrations due to the moving loads. However, a common view is that squealing is, above all, the result of selfsustained vibrations of the brake system [7]. There are mainly two types of instability that can lead to self-sustained vibrations. Both are due to the non-conservative nature of the friction forces. The first highlighted cause of instability is the possible decrease of the friction coefficient with the sliding speed [8, 9]. However, most brake squeal models are based on a second instability mechanism which is often called 'mode coupling' [10, 11, 12, 13, 14]. Geometric instabilities or kinematically constrained instabilities are the other employed names for this mechanism. This may occur with a constant friction coefficient, as defined by Coulomb's law, which couples normal and tangential forces at the interface.

In order to determine the stability of a dynamical system, a classical method consists in solving the eigenvalue problem corresponding to the system linearized around the equilibrium [10]. This simple technique provides complex modes and complex eigenvalues. Eigenvalues with positive real parts are characteristics of unstable equilibrium points which diverge from steady sliding and lead to self-sustained vibrations. Many studies are limited to this approach and do not explicitly calculate the self-sustained vibrations. Indeed, a pure non-linear transient or stationary analysis is necessary to compute the limit cycles the problem tends to [15].

When finite element models are considered, computing the stability requires to solve a non-symmetrical eigenvalue problem with a great number of degrees of freedom. Such a problem can be solved by using widespread iterative 


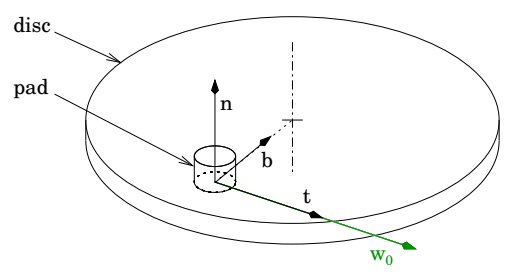

Figure 1: Local cartesian coordinate system at one particular point of the interface

algorithms based on Arnoldi process [16] or less known techniques such as the residue iterative method [17]. In any case, the use of iterative methods is highly expensive. To overcome this computational problem, a common technique is to expand the dynamic equations on reduced real bases. Practically, bases composed of frictionless (real) coupled modes or merely free components modes are often used. However, the question of errors provided by the reduction is rarely investigated.

In this context, Component Mode Synthesis (CMS) [18, 19, 20, 21, 22] appears to be a logical issue for the dynamical study of a brake mechanism composed of many substructures. It offers the advantage of computing component modes separately, thus requiring less computational efforts than directly calculating the global modes. A key point in CMS techniques is that the reduced bases not only contain components' normal modes but also some static boundary modes, such as attachments modes in free-interface methods or constrained modes in fixed-interface ones [22]. These modes allow statically complete bases to be built which greatly improve the results.

In this paper, the performances of reduced bases are investigated in the case of a simple disc/pads system. Bases composed of real coupled modes and bases provided by CMS techniques are tested. An enrichment of these bases is proposed in order to improve the precision of the results. The idea behind this enrichment is to build bases statically complete even when friction forces are taken into account. In particular, new rubbing attachments modes are proposed to adapt free-interface CMS techniques to frictional contact. In addition, when real coupled modes are used, it is suggested to complete the basis by the static response to a first-order approximation of the friction forces.

In section 2, the formulation of the problem is described, based on the previous work initially proposed by Moirot [23] and used for instance by Lorang [14]. Reduction principle and errors criteria which are employed to evaluate the reduction approximation are given in section 3. Section 4 introduces the disc brake model. Components modes and real coupled modes are analyzed and a preliminary stability study is performed. Classical CMS bases are first applied on the frictionless problem in section 5. Finally, in section 6, enrichments are introduced for the problem with friction and performances of classical and enriched bases are compared and discussed.

\section{Formulation of the problem}

In this section, the discretized equations governing the stability of the steady sliding of a disc/pads system are derived. The main assumptions are the linear elasticity in the volumes of the structures combined with unilateral contact and Coulomb friction at the interface. At each point of the interface, a local cartesian coordinate system $(\underline{n}, \underline{t}, \underline{b})$ is defined where $\underline{n}$ is the direction normal to the disc, $\underline{t}$ is the direction of the steady sliding and $\underline{b}$ is the radial direction defined as $\underline{b}=\underline{n} \wedge \underline{t}$ (c.f. figure 1).

\subsection{Continuous non-linear form}

The rotation speed of the disc is supposed to be constant and sufficiently low so that the gyroscopic terms and the volume forces induced by rotation may be neglected. By using an eulerian description, the displacements field $\underline{u}$ and the stresses tensor $\underline{\underline{\sigma}}$ in the volumes $D$ and $P$ of the disc and the pads verify:

$$
\underline{\underline{\sigma}}=\underline{\underline{A}}: \nabla \underline{u}, \quad \nabla \cdot \underline{\underline{\sigma}}=\rho \underline{\ddot{u}} \quad \forall \underline{x} \in D, P
$$

where $\underline{\underline{A}}$ is Hook's tensor. 
On the surface $S_{c}$ potentially in contact, the disc and the pads are coupled with conditions of unilateral contact with Coulomb friction, which may be expressed by:

$$
\begin{aligned}
& R_{n} \leq 0, g \leq 0, g R_{n}=0 \forall \underline{x} \in S_{c} \\
&\left|\underline{R}_{t}\right| \leq-\mu R_{n},|\underline{w}| \neq 0 \Rightarrow \underline{R}_{t}=\mu R_{n} \frac{\underline{w}}{|\underline{w}|} \quad \forall \underline{x} \in S_{c}
\end{aligned}
$$

In these equations, $g=g_{0}+u_{n}$ denotes the normal gap between the disc and the pads where $g_{0}$ is the initial gap and $u_{n}$ is the relative normal dynamic displacement. In addition, $\underline{w}=w_{0} \underline{t}+\underline{\dot{u}}_{t}$ denotes the sliding velocity between the disc and the pads where $w_{0}$ is the stationary sliding velocity due to the disc rotation and $\underline{\dot{u}}_{t}$ is the relative tangential dynamic velocity. Finally, $R_{n}$ and $\underline{R}_{t}$ represent the normal and tangential contact forces between the disc and the pads. The friction coefficient $\mu$ is supposed to be constant.

\subsection{Continuous linear form}

If the rotation speed is not equal to zero, the previous equations may have a quasi-static solution, or steady sliding, for which $\underline{\dot{u}}=0$ (the existence and the uniqueness of this equilibrium is not discussed here). If initial conditions differ from the steady sliding, two cases may be distinguished: the stable — non-squealing — case if the solution converges to the equilibrium, and the unstable - squealing - case if the solution grows, leading to self-sustained vibrations. The analysis of the stability is generally achieved by a linearization method. Considering small regular sliding perturbations that do not break the contact, the contact forces may be linearized. On the contact surface $S_{0}$ in the quasi-static solution, the perturbation verifies the following equations:

$$
\begin{array}{rr}
u_{n}=0 & \forall \underline{x} \in S_{0} \\
\underline{R}_{t}=\mu R_{n} \underline{t}-c_{b} \dot{u}_{b} \underline{b} & \forall \underline{x} \in S_{0}
\end{array}
$$

In these equations, $\dot{u}_{b}=\underline{\dot{u}}_{t} \cdot \underline{b}$ denotes the relative tangential velocity between the disc and the pads in the direction $\underline{b}$ and $c_{b}=R_{n 0} / w_{0}$ where $R_{n 0}$ is the quasi-static normal contact force. In the expression of the linearized friction force, the first term (along $\underline{t}$ ) is classical and comes from the linearization of the module of the friction force. The second term (along $\underline{b}$ ) is less well-known and is due to the linearization of its direction; it only appears in three dimensional models and acts as a viscous contact damping (it is also referred to as radial dissipation effect by some authors [24]).

\subsection{Discrete linear form}

By using a finite element method with conforming linear meshes at the interface, the previous linear equations (1) and (3) may be written in a discrete form as follows :

$$
\begin{aligned}
\mathbf{M} \ddot{\mathbf{u}}+\mathbf{C}_{b} \dot{\mathbf{u}}+\mathbf{K u} & =\mathbf{P}_{n}^{T} \mathbf{r}_{n}+\mathbf{P}_{t}^{T} \mathbf{r}_{t} \\
\mathbf{P}_{n} \mathbf{u} & =0 \\
\mathbf{r}_{t} & =\mu \mathbf{r}_{n}
\end{aligned}
$$

where $\mathbf{M}$ and $\mathbf{K}$ denote the mass and stiffness matrices of the uncoupled system, $\mathbf{C}_{b}$ is the damping matrix associated to the viscous contact damping $c_{b}$ and $\mathbf{u}$ represents the corresponding displacements vector. In addition, $\mathbf{r}_{n}$ and $\mathbf{r}_{t}$ denote the normal and tangential reaction forces at the contact nodes along the directions $\underline{n}$ and $\underline{t}$ whereas $\mathbf{P}_{n}$ and $\mathbf{P}_{t}$ are the projection matrices on the relative displacements between the disc and the pads along the same directions. After some algebraic manipulations, all the linear contact constraints may be eliminated and system (4) may be written in a more compact form :

$$
\begin{gathered}
\mathbf{u}=\mathbf{T}_{c} \mathbf{u}_{c} \\
\tilde{\mathbf{M}}_{c} \ddot{\mathbf{u}}_{c}+\tilde{\mathbf{C}}_{c} \dot{\mathbf{u}}_{\mathbf{c}}+\tilde{\mathbf{K}}_{c} \mathbf{u}_{c}=0
\end{gathered}
$$


where

$$
\begin{aligned}
\tilde{\mathbf{M}}_{c}, & =\mathbf{T}_{c}^{T} \tilde{\mathbf{M}} \mathbf{T}_{c} \\
\tilde{\mathbf{K}}_{c} & =\mathbf{T}_{c}^{T} \tilde{\mathbf{K}} \mathbf{T}_{c} \\
\tilde{\mathbf{C}}_{c} & =\mathbf{T}_{c}^{T} \mathbf{C}_{b} \mathbf{T}_{c}
\end{aligned}
$$

and

$$
\begin{aligned}
\tilde{\mathbf{M}} & =\mathbf{M}+\mathbf{M}_{\mu} \\
\tilde{\mathbf{K}} & =\mathbf{K}+\mathbf{K}_{\mu}
\end{aligned}
$$

In the above expressions, $\mathbf{T}_{c}$ is a basis for the kernel of the normal contact constraints $\mathbf{P}_{n}, \mathbf{M}_{\mu}$ and $\mathbf{K}_{\mu}$ are nonsymmetrical matrices taking into account the effects of the friction forces and given by:

$$
\begin{aligned}
\mathbf{M}_{\mu} & =\frac{\mu}{2} \mathbf{P}_{t}^{T} \mathbf{P}_{n} \mathbf{M} \\
\mathbf{K}_{\mu} & =\frac{\mu}{2} \mathbf{P}_{t}^{T} \mathbf{P}_{n} \mathbf{K}
\end{aligned}
$$

\subsection{Complex eigenvalue analysis}

The stability of the steady sliding may then be deduced from a complex eigenvalue analysis of the system:

$$
\left(\lambda^{2} \tilde{\mathbf{M}}_{c}+\lambda \tilde{\mathbf{C}}_{c}+\tilde{\mathbf{K}}_{c}\right) \phi_{c}=0
$$

providing a set of complex modes $\phi_{i}=\mathbf{T}_{c} \phi_{c i}$ and complex eigenvalues $\lambda_{i}=R_{i}+i \omega_{i}$ in the frequency range of interest. The perturbation may be approximated by a truncated superposition of these modes, oscillating at their natural pulsation $\omega_{i}$ but with divergent or vanishing envelopes controlled by $R_{i}$ and initial complex amplitudes $a_{i}=\left|a_{i}\right| \exp \left(\varphi_{i}\right)$ provided by the initial conditions :

$$
\begin{aligned}
\mathbf{u} & \simeq \operatorname{Re}\left(\sum_{i} a_{i} \phi_{i} \exp \left(\lambda_{i} t\right)\right) \\
& \simeq \sum_{i}\left|a_{i}\right| \exp \left(R_{i} t\right)\left(\operatorname{Re}\left(\phi_{i}\right) \cos \left(\omega_{i} t+\varphi_{i}\right)-\operatorname{Im}\left(\phi_{i}\right) \sin \left(\omega_{i} t+\varphi_{i}\right)\right)
\end{aligned}
$$

If all the modes are stable (i.e. $\forall i R_{i}<0$ ) the perturbation vanishes. On the other hand, if a complex mode is unstable (i.e. $\exists i R_{i}>0$ ), the perturbation grows, leading to self-sustained vibrations. This case may happen with friction forces because of the presence of the non-symmetric matrices $\mathbf{M}_{\mu}$ and $\mathbf{K}_{\mu}$. For each mode, a divergence rate may also be defined, physically equivalent to a negative damping ratio :

$$
\xi_{i}=\frac{R_{i}}{\omega_{i}}
$$

$R_{i}\left(\right.$ unit s$\left.^{-1}\right)$ corresponds to the geometric divergence of the envelope of mode $i$, whereas divergence rate $\xi_{i}$ unit rad $^{-1}$ ) corresponds to that geometric divergence in phase unit.

\section{Reduction of the problem}

\subsection{Principle}

Solving a large non-symmetric eigenvalue problem like system (9) is very expensive from a computational point of view. Iterative algorithms are available but computation times are often prohibitive to conducting parameter studies. 
Consequently, Ritz reduction methods are often used to solve the problem. This consists in searching for the best approximated solution in a reduced subspace characterized by a basis $T_{r}$, by solving the reduced eigenvalue problem:

$$
\left(\lambda^{2} \tilde{\mathbf{M}}_{r}+\lambda \tilde{\mathbf{C}}_{r}+\tilde{\mathbf{K}}_{r}\right) \phi_{r}=0
$$

where

$$
\begin{aligned}
\tilde{\mathbf{M}}_{r} & =\mathbf{T}_{r}^{T} \tilde{\mathbf{M}} \mathbf{T}_{r} \\
\tilde{\mathbf{K}}_{r} & =\mathbf{T}_{r}^{T} \tilde{\mathbf{K}} \mathbf{T}_{r} \\
\tilde{\mathbf{C}}_{r} & =\mathbf{T}_{r}^{T} \mathbf{C}_{b} \mathbf{T}_{r}
\end{aligned}
$$

providing a set of approximated complex modes $\left\{\hat{\phi}_{i}\right\}=\mathbf{T}_{r} \phi_{r i}$ and approximated complex eigenvalues $\hat{\lambda}_{i}=\hat{R}_{i}+i \hat{\omega}_{i}$ in the frequency range of interest. Obviously, to be compatible, vectors in the reduced subspace must at least verify the normal contact constraints, i.e. $\mathbf{P}_{n} \mathbf{T}_{r}=\mathbf{0}$.

A widely used method is to choose the real coupled modes (without friction) of the structure as a reduced basis. Indeed, these modes are calculated by solving a real symmetric eigenvalue problem, for which very efficient iterative algorithms are available. Another used method is to build a reduced basis from component modes, according to the so-called Component Mode Synthesis. These bases may give good results in some cases. However, as shown in the last section of the paper, a better approximation is obtained by using bases which take into account particular static responses to friction forces.

\subsection{Quality of the approximation}

First, the criteria employed to evaluate the quality of the approximation due to the reduction are presented below.

\subsubsection{Error on the residues}

A first criterion is defined by considering the force residue of the non-reduced eigenvalue equation for a couple of approximated mode $\hat{\phi}_{i}$ and approximated eigenvalue $\hat{\lambda}_{i}$ :

$$
\mathbf{R}_{i}^{F}=\mathbf{T}_{c}^{T}\left(\hat{\lambda}_{i}^{2} \tilde{\mathbf{M}}+\hat{\lambda}_{i} \mathbf{C}_{b}+\tilde{\mathbf{K}}\right) \hat{\phi}_{i}
$$

where the matrix $\mathbf{T}_{c}$ is needed here to eliminate the normal reaction forces at the interface. The method proposed by Bobillot is then used [17]: in order to obtain an energy indicator, the force residue is first converted into a displacement residue by using the stiffness matrix of the frictionless coupled system $\mathbf{K}_{c}=\mathbf{T}_{c}^{T} \mathbf{K} \mathbf{T}_{c}$ :

$$
\mathbf{R}_{i}^{U}=\mathbf{K}_{c}^{-1} \mathbf{R}_{i}^{F}
$$

Finally, the residue error is defined as the ratio between the deformation energy of the residue and the deformation energy of the considered mode :

$$
\varepsilon_{i}^{\mathrm{res}}=\frac{\left|\mathbf{R}_{i}^{U^{* T}} \mathbf{R}_{i}^{F}\right|}{\hat{\phi}_{i}^{* T} \mathbf{K} \hat{\phi}_{i}}
$$

\subsubsection{Error on the mode shapes}

Another criterion is defined by comparing the approximated complex modes with the exact complex modes. For a given exact mode $\phi_{i}$, the first step is to identify the corresponding approximated complex mode. This is achieved by reordering the approximated base such that:

$$
\operatorname{MAC}-\mathrm{M}\left(\phi_{i}, \hat{\phi}_{i}\right)=\max _{j}\left(\operatorname{MAC}-\mathrm{M}\left(\phi_{i}, \hat{\phi}_{j}\right)\right)
$$



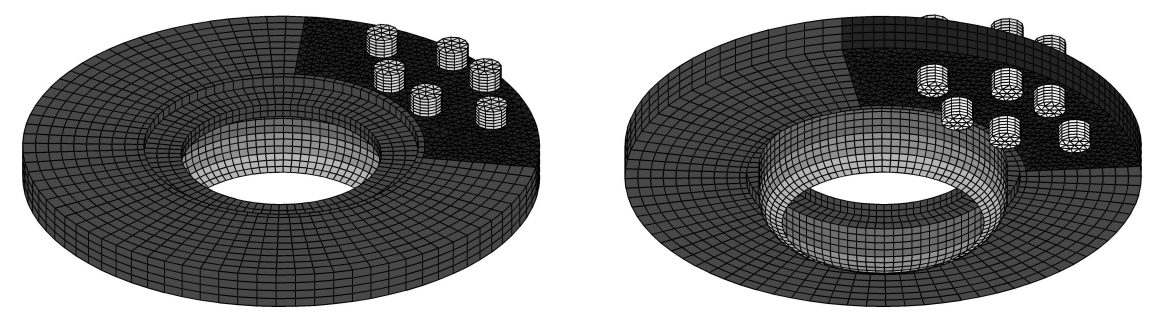

Figure 2: The disc/pads finite element model

where MAC-M is the complex mass-weighted Modal Assurance Criterion defined by:

$$
\operatorname{MAC}-\mathbf{M}\left(\phi_{i}, \hat{\phi}_{j}\right)=\frac{\left|\phi_{i}^{* T} \mathbf{M} \hat{\phi}_{j}\right|^{2}}{\left|\phi_{i}^{* T} \mathbf{M} \phi_{i}\right|\left|\hat{\phi}_{j}^{* T} \mathbf{M} \hat{\phi}_{j}\right|}
$$

This criterion was already defined for real modes as the NOC [25]. Thanks to this reordering, $\hat{\phi}_{i}$ is the closest approximated mode to $\phi_{i}$ by considering the classical complex norm and scalar product associated with the frictionless mass matrix. The error on the mode shapes is then defined as:

$$
\varepsilon_{i}^{\mathrm{mac}}=1-\operatorname{MAC}-\mathrm{M}\left(\phi_{i}, \hat{\phi}_{i}\right)
$$

No criterion is defined on the extra diagonal terms of the MAC-M since complex modes are not supposed to be orthogonal with each other. Indeed, no hermitian scalar product may be associated with the complex eigenvalue problem because of the non-symmetry induced by the friction forces.

\subsubsection{Error on the frequencies and divergence rates}

The matching of the approximated and exact bases now allows the calculation of some errors on frequencies:

$$
\varepsilon_{i}^{\mathrm{frq}}=\frac{\left|\omega_{i}-\hat{\omega}_{i}\right|}{\omega_{i}}
$$

and also on divergence rates:

$$
\varepsilon_{i}^{\text {div }}=\left|\xi_{i}-\hat{\xi}_{i}\right|
$$

where $\xi_{i}$ is the exact divergence rate and $\hat{\xi}_{i}$ the approximated divergence rate. This last criterion is defined as an absolute error because the divergence rates are relative indicators themselves. Practically, in order to make any conclusions on stability, this error has to be compared with the precision of a damping ratio measurement.

\section{The disc/pads system}

In this section, the model used to evaluate the performance of some reduced bases is described. This simple model (cf. figure 2) is derived from a more complex one which was developed to find instabilities in a TGV (French highspeed train) braking system $[14,26,27,28]$. The main characteristic of this system is the geometry of the pads and in particular the friction material which is distributed into a set of small cylindrical pins. Compared with the original model, several simplifications have been performed in order to reduce its size. Firstly, the flexibility of the pads' supporting structures (backplate and support) is neglected: a fixed boundary condition is assumed for the external face of the cylindrical pins. Secondly, only 6 pins applied on either sides of the disc are taken into account (vs 18 for the original model). Lastly, a rougher mesh is used since the frequency range of interest is restrained here to $0-5000$ $\mathrm{Hz}$ which leads to a total number of degrees of freedom $n=30942$ and a number of contact nodes $n_{c}=228$. This 


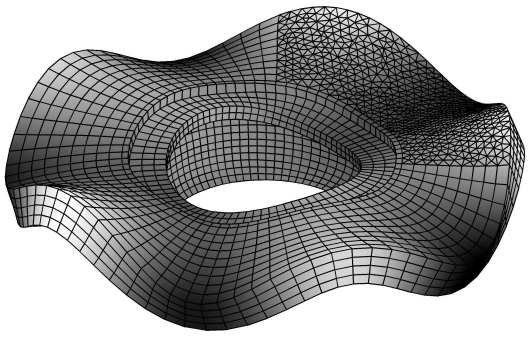

(a)

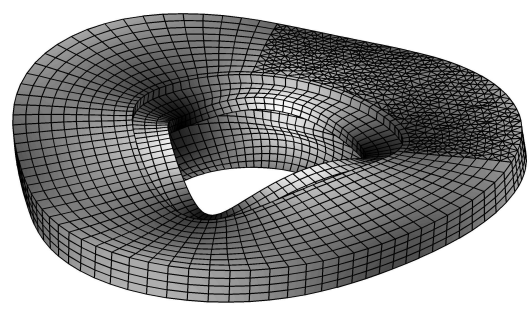

(c)

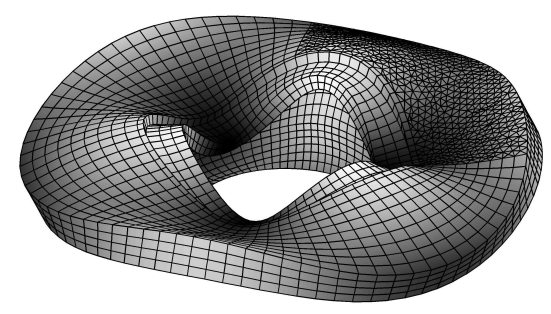

(b)

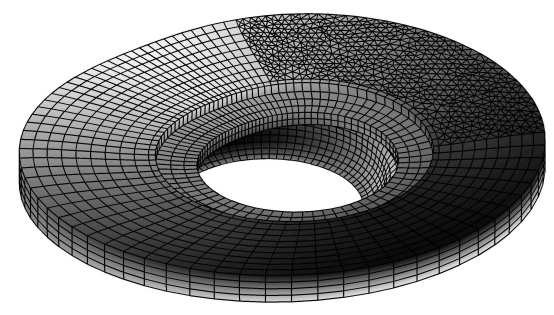

(d)

Figure 3: Free disc modes : (a) A0-5; (b) A1-3; (c) R0-3; and, (d) C0-1

simplified model is sufficient to bring out instabilities quite similar to those obtained with the original model and representative of some of the squealing noises generated on the trains.

\subsection{Component modes}

In a first step, the modes of each component are determined separately in order to go further into their own dynamics. For the disc with free boundary conditions on the contact surface, three mode types are distinguished:

- the axial modes Am- $n$ for which axial deformations are dominant (out-of-plane, flexion);

- the radial modes $\mathrm{R} m-n$ for which radial deformations are dominant (in-plane);

- the circumferential modes $\mathrm{C} m-n$ for which circumferential deformations are dominant (in-plane).

In addition, each mode is characterized by a number of nodal circles $m$ and a number of nodal diameters $n$. Because of the axi-symmetry of the disc, all modes with one nodal diameter or more are double, which means that there are two modes at the same frequency with the same shape but shifted by an angle equal to $\pi /(2 n)$. Some examples of the different encountered modes are represented on figure 3 .

Pins modes are computed for 'sliding' boundary conditions on the contact surface that are fixed in the normal direction and free in the tangential one. With these conditions, the dynamic behavior of the pins is rather close to the one which takes place in coupled conditions since the disc is much more rigid than the pins. Only three modes per pin are found below $5 \mathrm{kHz}$ : a double flexural mode F1 and a torsional mode T1 (cf. figure 4).

\subsection{Real coupled modes}

The second step is the determination of the real coupled modes (without friction i.e. $\mathbf{M}_{\mu}=\mathbf{K}_{\mu}=\mathbf{C}_{b}=\mathbf{0}$ ) for which the disc and the pins are coupled only through normal degrees of freedom on the contact surface. They are computed here by using a classical IRA/Sorensen iterative algorithm [29]. In the frequency range above $1500 \mathrm{~Hz}$, these coupled modes are easy to describe. One may distinguish the modes controlled by the disc from the modes controlled by the 


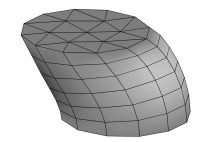

(a)

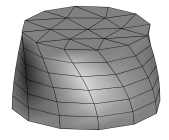

(b)

Figure 4: 'Sliding' pin modes: (a) F1; and, (b) T1

\begin{tabular}{lc|cccccc} 
& & Comp. modes & Real coupled modes & \multicolumn{2}{c}{ Complex modes $\mathbf{C}_{b}=\mathbf{0}$} & \multicolumn{2}{c}{ Complex modes $\mathbf{C}_{b} \neq \mathbf{0}$} \\
Type & & Freq. [Hz] & Frequency [Hz] & Freq. [Hz] & Div. [\%] & Freq. [Hz] & Div. [\%] \\
\hline A0-1 & $(-/+)$ & 251 & $295 / 312$ & $295 / 312$ & - & $296 / 313$ & - \\
A0-0 & & 352 & & & & & \\
A0-2 & $(-/+)$ & 569 & $489 / 697$ & $489 / 700$ & - & $489 / 700$ & - \\
C0-0 & & 644 & 644 & 645 & - & 644 & - \\
R0-1 & $(-/+)$ & 649 & $643 / 647$ & $644 / 648$ & - & $644 / 648$ & $-/-0.093$ \\
$?$ & & & 1057 & 1061 & - & 1061 & - \\
A0-3 & $(-/+)$ & 1267 & $1374 / 1567$ & $1379 / 1576$ & - & $1379 / 1576$ & - \\
A1-0 & & 1404 & 1449 & 1450 & - & 1450 & - \\
R0-2 & $(-/+)$ & 1822 & $1823 / 1825$ & $1823 / 1826$ & - & $1823 / 1826$ & - \\
A1-1 & $(-/+)$ & 2082 & $2087 / 2150$ & $2087 / 2153$ & - & $2087 / 2153$ & - \\
A0-4 & $(-/+)$ & 2220 & $2284 / 2305$ & 2297 & \pm 0.64 & 2297 & $+0.65 /-0.67$ \\
A0-5 & $(-/+)$ & 3345 & $3380 / 3392$ & 3376 & \pm 0.98 & $3376 / 3377$ & $-1.02 /+0.95$ \\
A1-2 & $(-/+)$ & 3365 & $3350 / 3371$ & $3352 / 3386$ & - & $3352 / 3387$ & $-/-0.078$ \\
& & & & & & 3521 & -0.12 \\
& & & & 3516 to 3564 & - & 3525 to 3528 & - \\
F1 & $(\times 24)$ & 3353 & 3551 to 3562 & 3542 & \pm 0.89 & 3535 & -0.51 \\
& & & & 3550 & \pm 0.10 & 3537 & +0.88 \\
& & & & & & 3552 to 3563 & -1.76 to -1.07 \\
R0-0 & & 3704 & 3704 & 3704 & - & 3704 & - \\
T1 & $(\times 12)$ & 3934 & 3934 & 3934 & - & 3933 & -2.34 to -1.41 \\
R0-3 & $(-/+)$ & 3990 & $3989 / 3991$ & 3989 & - & $3989 / 3990$ & - \\
C0-1 & $(-/+)$ & 4500 & $4499 / 4500$ & $4499 / 4500$ & - & $4499 / 4500$ & - \\
A0-6 & $(-/+)$ & 4659 & $4641 / 4653$ & $4641 / 4652$ & - & $4641 / 4652$ & - \\
A1-3 & $(-/+)$ & 4794 & $4800 / 4815$ & $4799 / 4814$ & - & $4800 / 4815$ & -
\end{tabular}

Table 1: Characteristics of the 66 first components modes, real coupled modes and complex modes for $\mu=0.4$ and $\mathbf{C}_{b}=\mathbf{0}$ or $\mathbf{C}_{b} \neq \mathbf{0}$ (- : nil or negligible) 


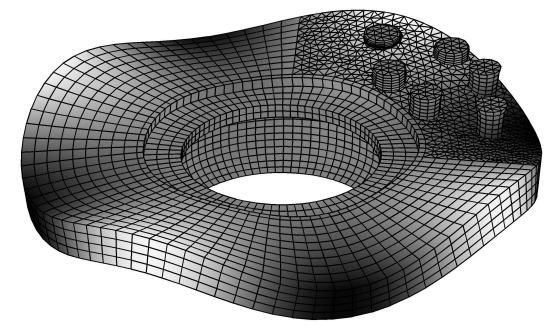

(a)

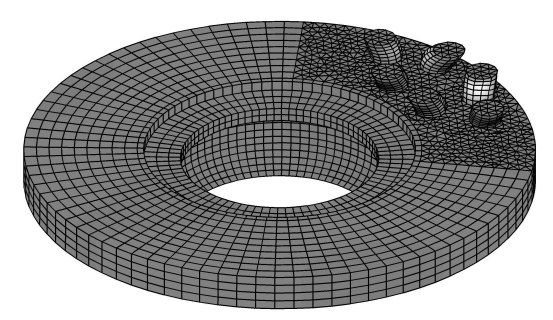

(c)

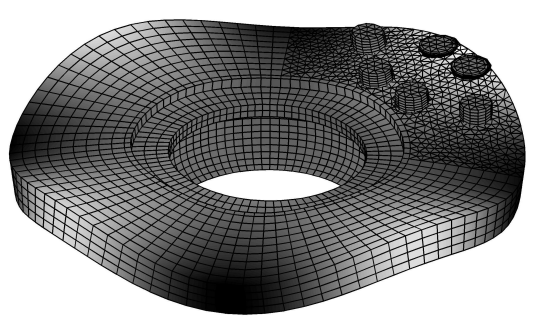

(b)

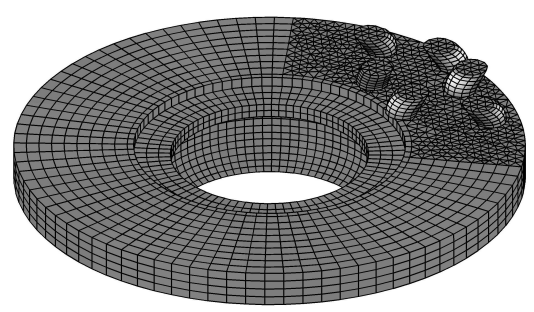

(d)

Figure 5: Real coupled modes: (a) A04-, (b) A04+ and (c) and (d) two F1-type

pins. The first ones are close to the modes of the free disc presented above but with pins deformations following the disc motion, whereas the other ones are very similar to the sliding pin modes F1 and T1 but with a little disc contribution (cf. figure 5). In the low-frequency range, the higher stiffness brought by the pins tends to modify more strongly the free disc modes and some of them are harder to describe. However, a designation based on components modes is kept for most of the coupled modes in all the frequency range (cf. table 1).

It is important to notice that, as the coupling breaks the symmetry of the components, disc double modes are split and pins multiples modes are dispersed. In particular, the difference between the frequencies of two disc modes of the same type (called - and +) may be explained by the difference of global stiffness brought by the pins according to the position of the disc nodal diameters. This is very pronounced for the axial disc modes and flexural pins modes. On the other hand, coupling has very little effect or no effect at all on some component modes with small normal contact amplitude such as the in-plane disc modes (circumferential and radial) and torsional pins modes.

\subsection{Complex modes}

The stability of the system is studied here for 90 values of the friction coefficient $\mu$, varying from 0 to 0.45 , by using the residue iterative method [17]. Two cases are considered by taking the damping matrix $\mathbf{C}_{b}$ into account or not ; note that no structural damping is taken into account in this paper. This matrix is the only one which depends on the equilibrium and thus is sensitive to the braking conditions. In this study, it is determined for a rotation speed equal to $4 \mathrm{rad} / \mathrm{s}$ and a static global load of $1000 \mathrm{~N}$ on each side of the disc. Neglecting it amounts to considering a high speed/low effort configuration. In both cases, the obtained complex modes are close to the real coupled modes in terms of frequencies and shapes except that some modes become unstable whereas others are damped.

Stability for $\mu=0.4$

When matrix $\mathbf{C}_{b}$ is neglected, the system has four unstable modes, two disc modes and two pins modes (cf. table 1). The main characteristic of the unstable modes is that they are not stationary: they rather look like rotating waves. Moreover, they work in pair with a similar stable mode, rotating the opposite way with a divergence rate of the same magnitude but the opposite sign. Adding damping matrix $\mathbf{C}_{b}$, while there is no structural damping, has a 


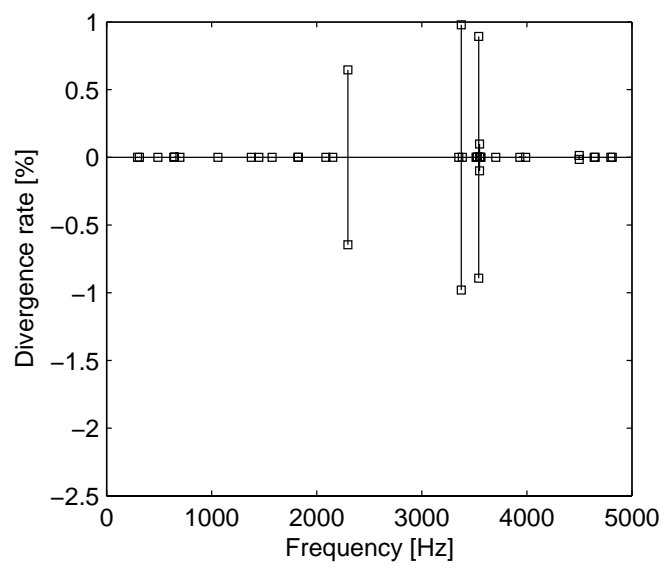

(a)

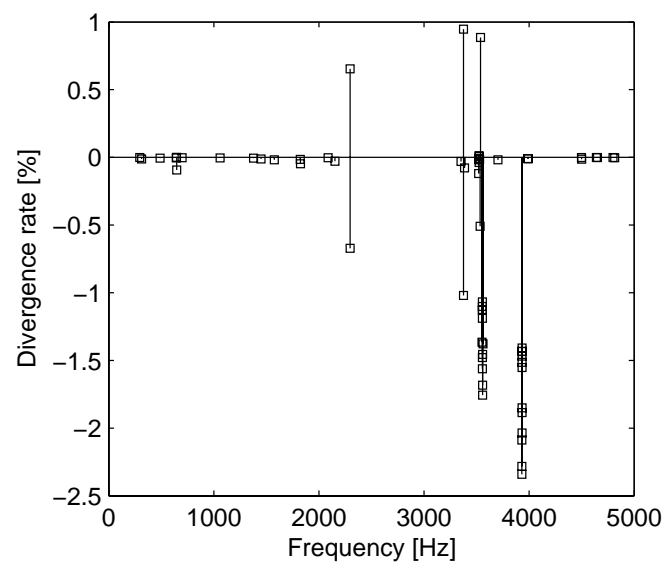

(b)

Figure 6: Stability charts for $\mu=0.4$ and: (a) $\mathbf{C}_{b}=0$; or (b) $\mathbf{C}_{b} \neq 0$

stabilizing effect. It breaks the symmetry of the stability chart (cf. figure 6) by reducing the divergence rates. As a consequence, the second slightly unstable pins mode becomes stable and many pins modes turn to highly negative divergence rates. It has been demonstrated that increasing damping does not always have a stabilizing effect [10]. The system is stabilized by a "lowering effect" when the two modes involved in the coalescence are equally damped. On the contrary, both "lowering" and "smoothing" effects take place when the two modes are not equally damped; in the case of a prevailing "smoothing effect" the system may be unintuitively destabilized by added damping. However the influence of damping upon stability is beyond the scope of this paper.

\section{Bifurcation}

Observing the evolution of the unstable modes with varying friction coefficient is useful to clarify the origin of instabilities. When neglecting $\mathbf{C}_{b}$, the coalescence of the disc modes is easy to observe (cf. figure 7): the frequencies of the two similar disc modes, distinct at $\mu=0$, become equal with increasing friction coefficient. There are then two modes at the same frequency, one being stable and the other unstable. The same coalescence phenomenon occurs for pins modes but the higher modal density makes it a bit more difficult to see (cf. figure 8). With increasing friction coefficient, an initially unstable pin mode restabilizes for $\mu=1.8$. When adding $\mathbf{C}_{b}$, rather similar results can be observed (cf. figure 7 and figure 9). Computing these graphs is extremely expensive: a complex eigenvalue problem has to be iteratively solved for each friction coefficient step. A reduced basis could therefore be useful to reduce the computation times.

\section{Reduced bases for the frictionless problem}

Before using reduced bases for the stability study, component mode synthesis methods (CMS) are tested on the problem without friction. The objective is to evaluate the performance of some bases constructed from the component modes for the calculation of the real coupled modes. This is a classical problem but it is presented here as a preliminary study and also as an introduction to the formalism used further in the stability study of section 6 .

\subsection{Description of the bases}

\subsubsection{Reduction of the components}

For the reduction of the components, two classical CMS bases are used. The first one is a combination of dynamic free-interface normal modes and static boundary attachments modes [19, 20, 21] whereas the second one uses fixedinterface dynamic normal modes plus static boundary constrained modes (original Craig-Bampton method [18]). In both cases, static boundary modes are added to dynamic normal modes in order to make the bases statically complete 


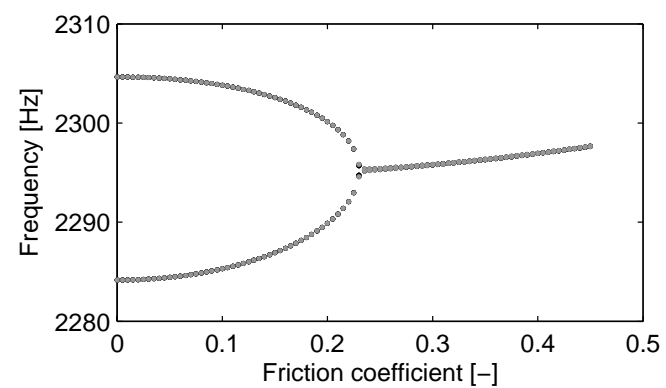

(a)

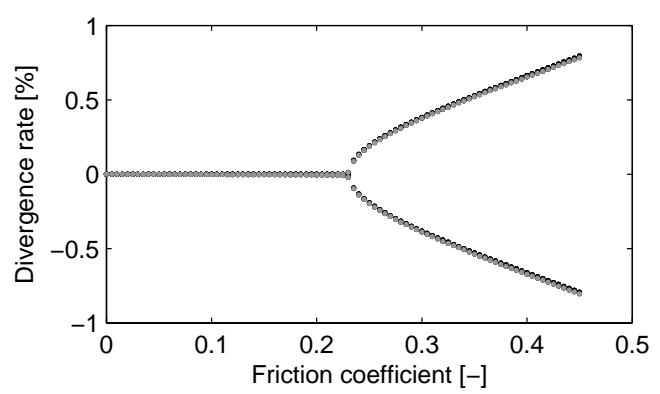

(c)

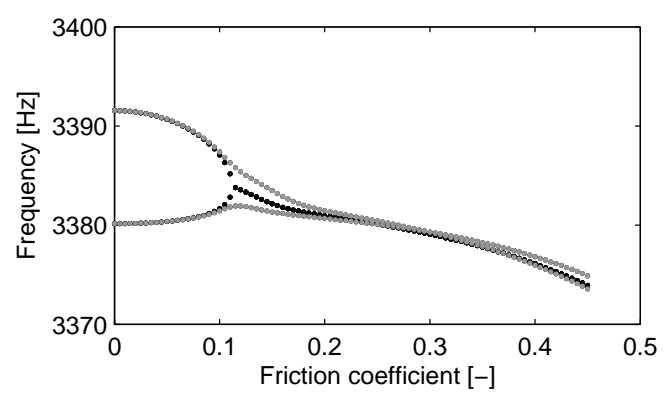

(b)

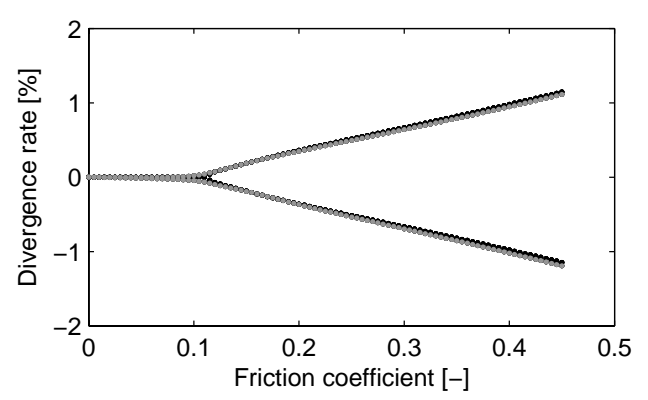

(d)

Figure 7: Coalescence of complex disc modes: (a)-(c) A04-/A04+; and, (b)-(d) A05-/A05+; black dots: $\mathbf{C}_{b}=\mathbf{0}$; gray dots: $\mathbf{C}_{b} \neq \mathbf{0}$

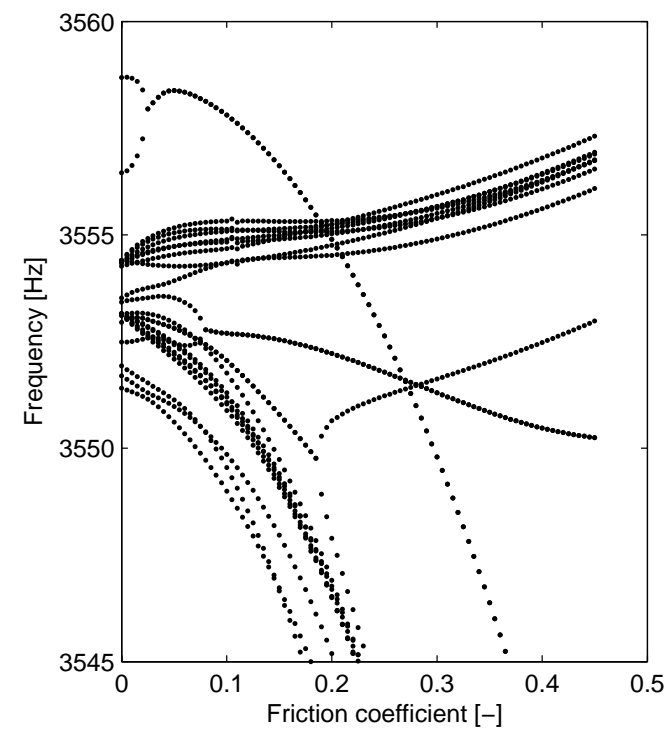

(a)

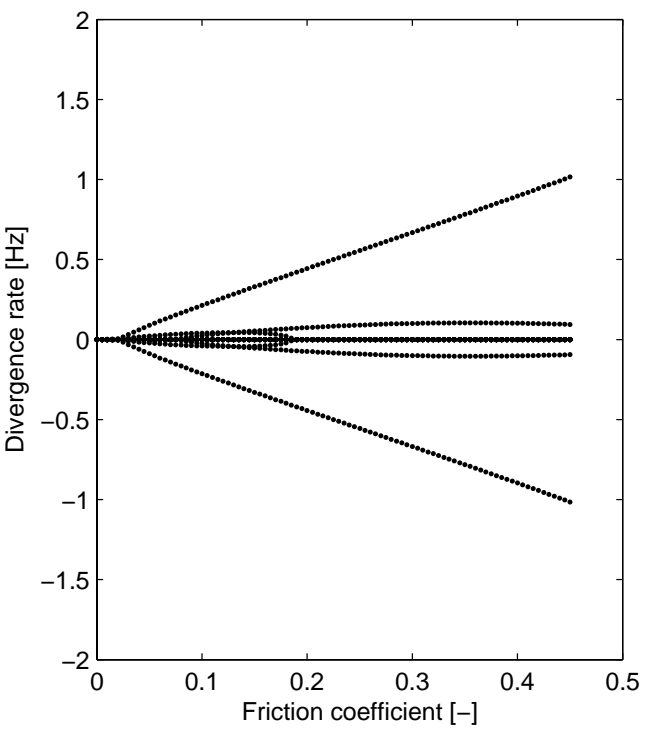

(b)

Figure 8: Coalescence of complex pins modes F1, $\mathbf{C}_{b}=\mathbf{0}$ : (a) frequency; (b) divergence rate 


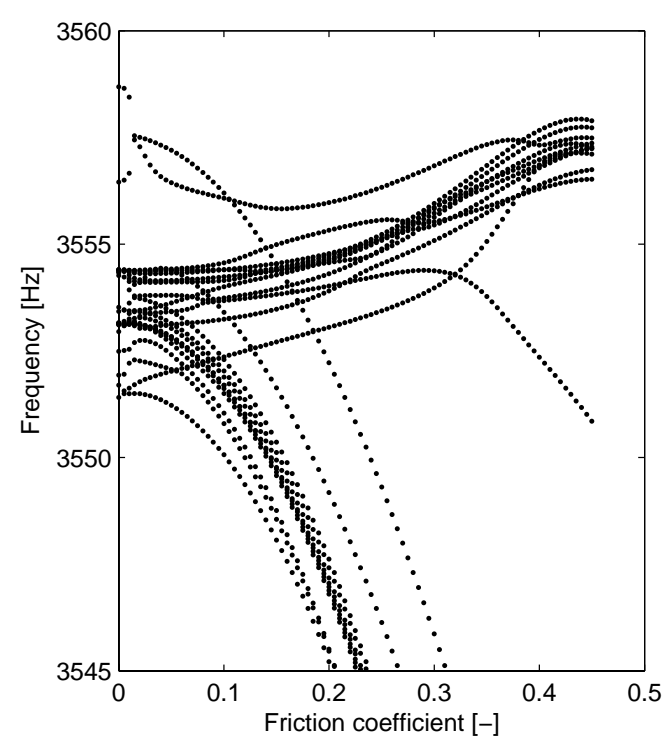

(a)

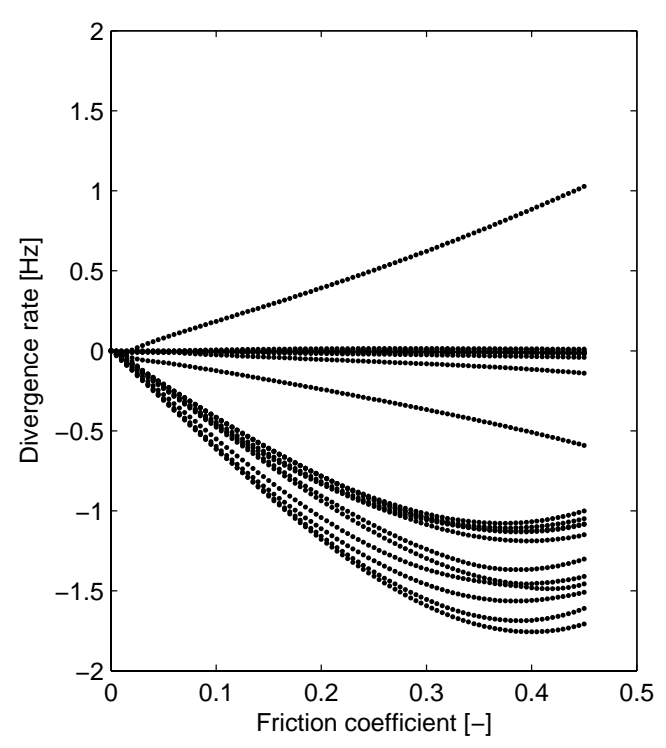

(b)

Figure 9: Coalescence of complex pins modes $\mathrm{F} 1, \mathbf{C}_{b} \neq \mathbf{0}$ : (a) frequency; (b) divergence rate

for the coupled system. In our problem, two components are considered (the disc and the whole set of pins) and the interface is restrained to normal degrees of freedom of the contact surface. In addition, since the disc and the pins have no rigid body modes, the definitions of the static boundary modes do not present any particular problem. If one then defines:

- the matrix $\boldsymbol{\Phi}_{f}^{D}$ (resp. $\boldsymbol{\Phi}_{f}^{P}$ ) of the $n_{m}^{D}$ (resp. $n_{m}^{P}$ ) first normal modes of the disc (resp. pins) with free boundary conditions on contact nodes,

- the matrix $\boldsymbol{\Phi}_{s}^{D}$ (resp. $\boldsymbol{\Phi}_{s}^{P}$ ) of the $n_{m}^{D}$ (resp. $n_{m}^{P}$ ) first normal modes of the disc (resp. pins) with sliding boundary conditions on contact nodes i.e. fixed in the normal direction and free in the tangential one,

- the matrix $\boldsymbol{\Psi}_{a n}^{D}$ (resp. $\boldsymbol{\Psi}_{a n}^{P}$ ) of the $n_{c}$ normal attachments modes of the disc (resp. pins) i.e. the static responses of the disc (resp. pins) to imposed unit normal forces on contact nodes,

- the matrix $\boldsymbol{\Psi}_{c n}^{D}$ (resp. $\boldsymbol{\Psi}_{c n}^{P}$ ) of the $n_{c}$ normal constrained modes of the disc (resp. pins) i.e. the static responses of the disc (resp. pins) to imposed unit normal displacements on contact nodes,

the disc (resp. pins) free-interface complete base may be expressed as $\boldsymbol{\Phi}_{f}^{D} \boldsymbol{\Psi}_{a n}^{D}$ (resp. $\boldsymbol{\Phi}_{f}^{P} \boldsymbol{\Psi}_{a n}^{P}$ ) and the disc (resp. pins) fixed-interface complete base may be written $\boldsymbol{\Phi}_{s}^{D} \boldsymbol{\Psi}_{c n}^{D}\left(\operatorname{resp} . \boldsymbol{\Phi}_{s}^{P} \boldsymbol{\Psi}_{c n}^{P}\right)$. In our notations, all the modes are defined for the same set of degrees of freedom corresponding to the global displacement vector $\mathbf{u}$.

\subsubsection{Connection of the components}

Considering the two kinds of component bases presented above, four bases may be defined for the whole structure according to the choice made for each component. In this study, only three of them are tested (see also table 2):

- the "free" one combines free-interface bases for both the disc and the pins ; in this particular case, a global set of static boundary attachments modes $\left(\boldsymbol{\Psi}_{a n}^{D}-\boldsymbol{\Psi}_{a n}^{P}\right)$ is used rather than one for each component; this is a frequently used free-interface CMS technique : it amounts to adding constraints on the generalized coordinates (representing static boundary forces) associated to the attachments modes [21, 22];

- the "fixed" one combines fixed-interface bases for both the disc and the pins; 


\begin{tabular}{l|ccc} 
Method & Reduced basis $\mathbf{T}_{r 0}$ & size of $\mathbf{T}_{r 0}$ & size of $\mathbf{T}_{r}$ \\
\hline free & $\boldsymbol{\Phi}_{f}^{D} \boldsymbol{\Phi}_{f}^{P}\left(\boldsymbol{\Psi}_{a n}^{D}-\boldsymbol{\Psi}_{a n}^{P}\right)$ & $n_{m}^{D}+n_{m}^{P}+n_{c}$ & $n_{m}^{D}+n_{m}^{P}$ \\
fixed & $\boldsymbol{\Phi}_{s}^{D} \boldsymbol{\Phi}_{s}^{P} \boldsymbol{\Psi}_{c n}^{D} \boldsymbol{\Psi}_{c n}^{P}$ & $n_{m}^{D}+n_{m}^{P}+2 n_{c}$ & $n_{m}^{D}+n_{m}^{P}+n_{c}$ \\
mixed & $\boldsymbol{\Phi}_{f}^{D} \boldsymbol{\Phi}_{s}^{P} \boldsymbol{\Psi}_{a n}^{D} \boldsymbol{\Psi}_{c n}^{P}$ & $n_{m}^{D}+n_{m}^{P}+2 n_{c}$ & $n_{m}^{D}+n_{m}^{P}+n_{c}$
\end{tabular}

Table 2: CMS bases for the frictionless problem

\begin{tabular}{r|c|cc}
$\begin{array}{r}\text { Max. } \\
\text { Frequency }\end{array}$ & $\begin{array}{c}n_{m}^{D} \\
\text { all methods }\end{array}$ & free method & $\begin{array}{c}n_{m}^{P} \\
\text { fixed and mixed method }\end{array}$ \\
\hline $6 \mathrm{kHz}$ & 30 & 36 & 36 \\
$7.5 \mathrm{kHz}$ & 60 & 72 & 36 \\
$10 \mathrm{kHz}$ & 80 & 120 & 60
\end{tabular}

Table 3: Number of components normal modes taken into account in CMS

- the "mixed" one combines a free-interface basis for the disc and a fixed-interface basis for the pins.

Actually, in the mixed basis, the component normal modes presented in section 4 are used. It is thus expected to exhibit good results since the real coupled modes are close to these component modes. On the contrary, a mixed basis with a fixed-interface basis for the disc and a free-interface basis for the pins would be of poor interest since, in this case, component modes would be very far from the real coupled modes.

Before using the basis to solve the eigenvalue problem, the connection of the components is achieved by forcing the base to satisfy the normal contact constraints $\mathbf{P}_{n} \mathbf{T}_{r}=\mathbf{0}$. For an initial unconstrained reduced base $\mathbf{T}_{r 0}$, this is done by taking a final constrained reduced base $\mathbf{T}_{r}$ such that:

$$
\mathbf{T}_{r}=\mathbf{T}_{r 0} \mathbf{T}_{c r}
$$

where $\mathbf{T}_{c r}$ is a basis for the kernel of $\mathbf{P}_{n} \mathbf{T}_{r 0}$. It generally leads to the elimination of $n_{c}$ vectors in the basis which results in the bases sizes given in table 2 .

\subsection{Results}

In order to obtain good results with CMS, it is well known that component normal modes need to be computed in an extended frequency range compared with the one of interest. The three methods have thus been tested for three sets of normal modes corresponding to a maximum frequency equal to 1.2, 1.5 and 2 times the limit frequency of the study i.e. $6,7.5$ and $10 \mathrm{kHz}$. The numbers of disc and pins modes corresponding to these frequencies are given in table 3 . The errors due to the reduction are given on figures 10,11 and 12 . The error criteria on residues $\varepsilon_{i}^{\text {res }}$, frequencies $\varepsilon_{i}^{\text {frq }}$ and mode shapes $\varepsilon_{i}^{\mathrm{mac}}$ defined previously are used (which corresponds respectively to the res, frq and mac labels on the $\mathrm{x}$ axis of each figure). For the errors on frequencies and shapes, the reference modes are the real coupled modes presented in section 4 and computed by using a classical IRA/Sorensen algorithm. Only the maximum errors for each kind of mode (disc modes, F1 pin modes and T1 pin modes) are given.

For all tested bases, errors on torsional T1 pin modes are extremely small (out of scale on the figures) except for criterion $\varepsilon_{i}^{\mathrm{mac}}$. Actually, these modes have the same frequency and are not coupled with the disc: these are purely multiple modes. As a consequence, all linear combinations of them are solutions of the eigenvalue problem and a criterion based on individual shapes comparison is not pertinent in this particular case. For the other kinds of modes, results are coherent: errors corresponding to the three criteria move together in the same direction with the same order of magnitude. However, errors $\varepsilon_{i}^{\text {mac }}$ tend to reduce with more difficulty than the others.

The free basis gives rather good results, even in the $6 \mathrm{kHz}$ case, especially for the disc modes. This is not surprising since this is the disc which controls the dynamics of the whole system. When component modes up to $7.5 \mathrm{kHz}$ or $10 \mathrm{kHz}$ are kept, the errors are even divided by 100 for both the disc and pins modes and the free method reaches 


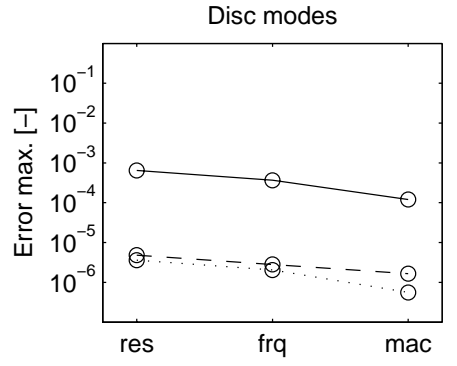

(a)

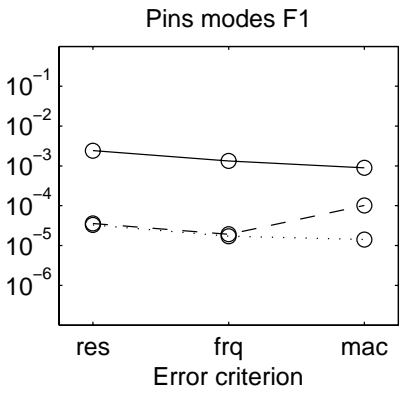

(b)

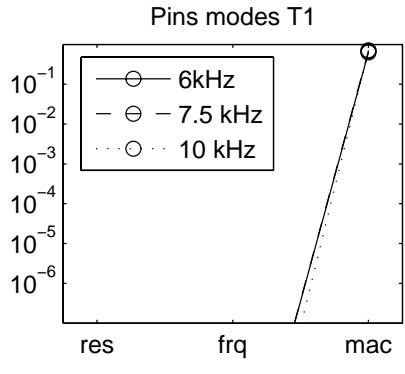

(c)

Figure 10: Performance of the free CMS basis for the frictionless problem for: (a) disc modes; (b) F1 pin modes; and, (c) T1 pin modes

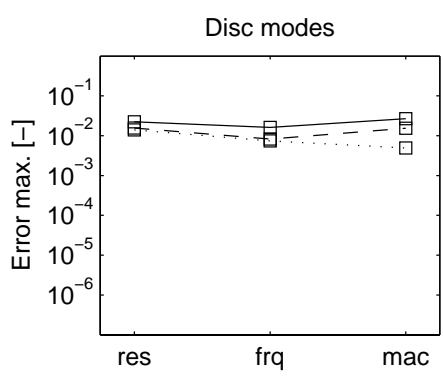

(a)

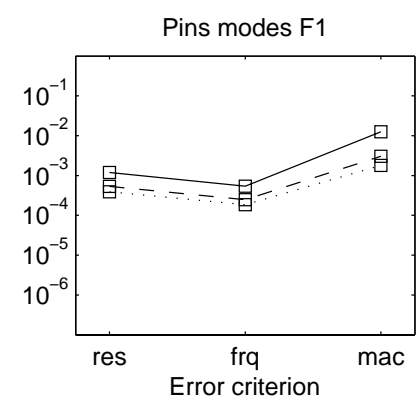

(b)

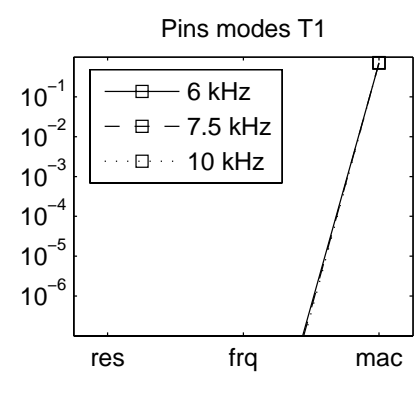

(c)

Figure 11: Performance of the fixed CMS basis for the frictionless problem for: (a) disc modes; (b) F1 pin modes; and, (c) T1 pin modes

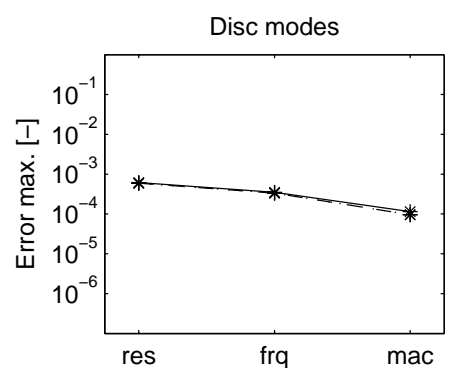

(a)

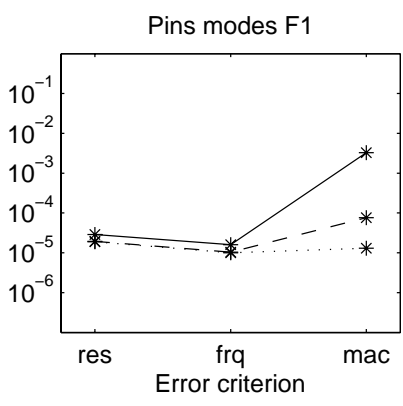

(b)

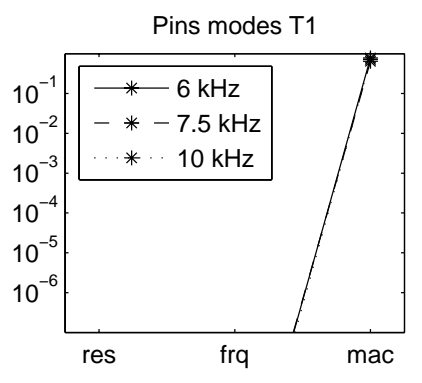

(c)

Figure 12: Performance of the mixed CMS basis for the frictionless problem for: (a) disc modes; (b) F1 pin modes; and, (c) T1 pin modes 


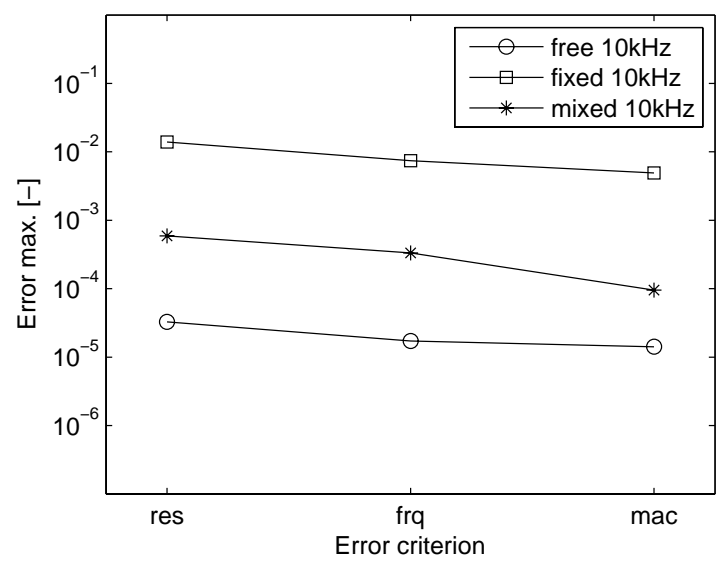

Figure 13: Global comparison of the performance of the CMS bases for the frictionless problem

its optimum. Conversely, the fixed basis is not adapted for a good description of the disc modes but gives acceptable results for the pins modes. However, keeping more components modes does not improve the performances of the basis in a significant way. As expected, the mixed basis gives very small errors for both the disc and the pins with a reduced number of component modes. However, as for the fixed method, the errors are not significantly reduced when the number of component modes increases. A global comparison of the three bases is given on figure 13. For this comparison, the maximum error on both the disc and F1 pins modes is taken into account and components modes up to $10 \mathrm{kHz}$ are kept. It is now clear that, despite the initial good performances of the mixed basis, the free one gives the best results when it has converged: the dynamics of the coupled system is better described when a sufficient number of free pins modes are taken into account.

\section{Reduced bases for the stability study}

In this section, the use of reduced bases for the stability analysis is investigated. The friction is taken into account in the eigenvalue problem leading to complex eigenvalues and complex modes. Two kinds of bases are tested and compared together:

- bases built from the components modes (CMS),

- bases built from the real coupled modes.

\subsection{Enrichment of CMS bases}

The CMS bases defined in the previous section and adapted to the frictionless problem may be used for the stability study. However, since friction is added, some forces apply on the tangential contact degrees of freedom in the coupled system. Therefore, it may be interesting to add some static responses to these forces in the bases through the use of tangential attachment modes. To that end, we define the matrix $\boldsymbol{\Psi}_{a t}^{D}$ (resp. $\boldsymbol{\Psi}_{a t}^{P}$ ) of the $n_{c}$ tangential attachments modes of the disc (resp. pins); it corresponds to the static responses of the disc (resp. pins) to imposed unit tangential forces on contact nodes in the direction $\underline{t}$. Thus, the enriched CMS bases given in table 4 may be constructed. An even more adapted approach is to include in the basis the static responses to normal and friction forces applied together on the components. This is done by defining some new rubbing attachment modes for the disc and the pins as:

$$
\begin{aligned}
& \boldsymbol{\Psi}_{a \mu}^{D}=\boldsymbol{\Psi}_{a n}^{D}+\mu \boldsymbol{\Psi}_{a t}^{D} \\
& \boldsymbol{\Psi}_{a \mu}^{P}=\boldsymbol{\Psi}_{a n}^{P}+\mu \boldsymbol{\Psi}_{a t}^{P}
\end{aligned}
$$




\begin{tabular}{l|ccc} 
Method & Reduced basis $\mathbf{T}_{r 0}$ & size of $\mathbf{T}_{r 0}$ & size of $\mathbf{T}_{r}$ \\
\hline Free + t.a.m. & $\boldsymbol{\Phi}_{f}^{D} \boldsymbol{\Phi}_{f}^{P}\left(\mathbf{\Psi}_{a n}^{D}-\boldsymbol{\Psi}_{a n}^{P}\right)\left(\boldsymbol{\Psi}_{a t}^{D}-\boldsymbol{\Psi}_{a t}^{P}\right)$ & $n_{m}^{D}+n_{m}^{P}+2 n_{c}$ & $n_{m}^{D}+n_{m}^{P}+n_{c}$ \\
Free with r.a.m. & $\boldsymbol{\Phi}_{f}^{D} \boldsymbol{\Phi}_{f}^{P}\left(\boldsymbol{\Psi}_{a \mu}^{D}-\boldsymbol{\Psi}_{a \mu}^{P}\right)$ & $n_{m}^{D}+n_{m}^{P}+n_{c}$ & $n_{m}^{D}+n_{m}^{P}$ \\
Fixed + t.a.m. & $\boldsymbol{\Phi}_{s}^{D} \boldsymbol{\Phi}_{s}^{P} \mathbf{\Psi}_{c n}^{D} \boldsymbol{\Psi}_{c n}^{P}\left(\mathbf{\Psi}_{a t}^{D}-\boldsymbol{\Psi}_{a t}^{P}\right)$ & $n_{m}^{D}+n_{m}^{P}+3 n_{c}$ & $n_{m}^{D}+n_{m}^{P}+2 n_{c}$ \\
Mixed + t.a.m. & $\boldsymbol{\Phi}_{f}^{D} \boldsymbol{\Phi}_{s}^{P} \boldsymbol{\Psi}_{a n}^{D} \boldsymbol{\Psi}_{c n}^{P}\left(\boldsymbol{\Psi}_{a t}^{D}-\boldsymbol{\Psi}_{a t}^{P}\right)$ & $n_{m}^{D}+n_{m}^{P}+3 n_{c}$ & $n_{m}^{D}+n_{m}^{P}+2 n_{c}$
\end{tabular}

Table 4: Enriched CMS bases for the stability study (t.a.m.: tangential attachment modes, r.a.m.: rubbing attachment modes)

\begin{tabular}{c|cc} 
Method & Reduced basis $\mathbf{T}_{r}$ & size of $\mathbf{T}_{r}$ \\
\hline Real & $\boldsymbol{\Phi}_{R}$ & $n_{R}$ \\
Real + t.a.m. & $\boldsymbol{\Phi}_{R}\left(\boldsymbol{\Psi}_{\text {cat }}^{D}-\boldsymbol{\Psi}_{c a t}^{P}\right)$ & $n_{R}+n_{c}$ \\
Real + 1st-ord. & $\boldsymbol{\Phi}_{R} \boldsymbol{\Psi}_{R \mu}$ & $2 n_{R}$
\end{tabular}

Table 5: Bases constructed from the real coupled modes for the stability study (t.a.m.: tangential attachment modes, $1^{\text {st }}$-ord.: $1^{\text {st }}$-order approximation of the response to frictional forces)

However, this enrichment technique may only apply when normal attachments modes are already present in the initial basis, which is the case for the free and the mixed CMS bases. In this paper, it has only been tested for the enrichment of the free CMS basis as it is summarized in table 4.

\subsection{Enrichment of the real coupled modes}

As previously mentioned, a widely used method is to choose the real coupled modes of the structure as a reduced basis. It is pertinent since efficient iterative algorithms are available for the resolution of the symmetric real problem. Thus, we define the matrix $\boldsymbol{\Phi}_{R}$ of the first $n_{R}$ normal modes of the frictionless coupled structure. Although this basis may give rather good results, it would be interesting to complete it by adding some static response to the friction forces, similar to the enrichment of the CMS bases. This is done by using the matrix $\boldsymbol{\Psi}_{c a t}^{D}$ (resp. $\boldsymbol{\Psi}_{c a t}^{P}$ ) of the $n_{c}$ tangential attachments modes of the coupled structure (with contact constraints but no friction). It corresponds to the static responses of the coupled structure to imposed unit tangential forces on disc (resp. pins) contact nodes in the direction $t$. With this technique, the basis is statically complete since the responses to every possible friction forces are included.

Another possibility is to carry out a first-order approximation of the friction forces and the corresponding static response. Although real coupled modes are obtained without friction, it is possible to calculate the static part of the friction forces which would be induced by the displacement field due to these modes:

$$
\mathbf{F}\left(\boldsymbol{\Phi}_{\mathbf{R}}\right)=\mathbf{K}_{\mu} \boldsymbol{\Phi}_{R}
$$

With the use of the stiffness matrix $\mathbf{K}_{c}$ of the frictionless coupled system, the corresponding static response is obtained:

$$
\boldsymbol{\Psi}_{R \mu}\left(\boldsymbol{\Phi}_{\mathbf{R}}\right)=\mathbf{T}_{c} \mathbf{K}_{c}^{-1} \mathbf{T}_{c}^{T} \mathbf{K}_{\mu} \mathbf{\Phi}_{R}
$$

Matrix $\boldsymbol{\Psi}_{R \mu}$ may then be used to complete the real coupled modes. This enrichment method finds its origin in the work of Plouin [30] where it has been proposed in a similar form for viscoelastic problems. The two resulting enriched bases and their size are summarized in table 5 . 


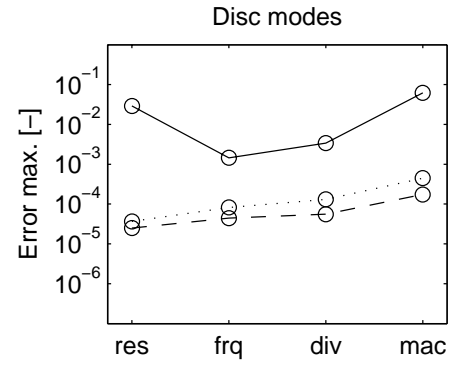

(a)

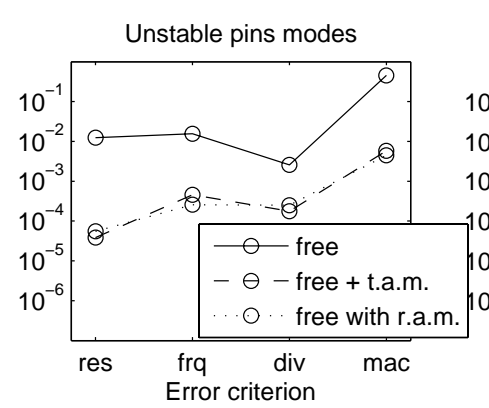

(b)

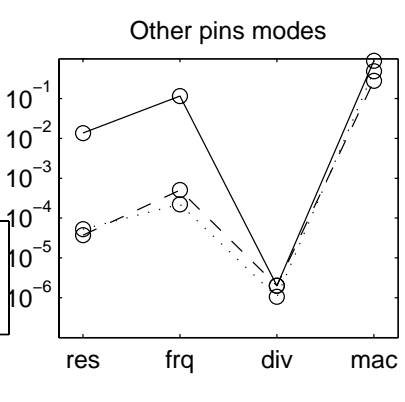

(c)

Figure 14: Performance of the free CMS bases for the stability study for: (a) disc modes; (b) unstable pins modes; and, (c) other pins modes

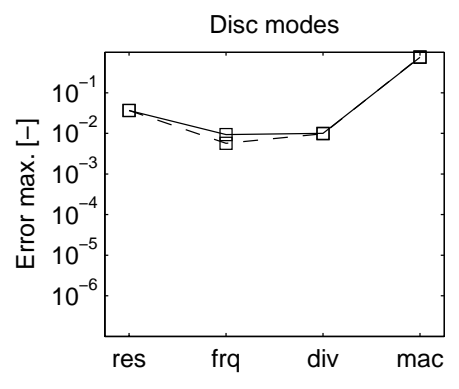

(a)

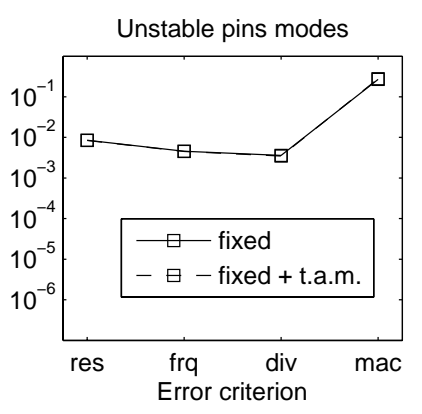

(b)

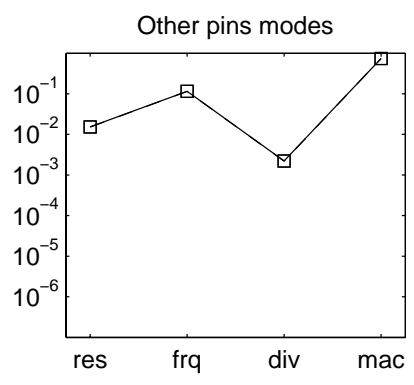

(c)

Figure 15: Performance of the fixed CMS bases for the stability study for: (a) disc modes; (b) unstable pins modes; and, (c) other pins modes

\subsection{Results for $\mu=0.4$}

The errors due to the reduction are given on figures 14,15 and 16 for the CMS bases and on figure 17 for the bases built from real coupled modes. The error criteria on residues $\varepsilon_{i}^{\text {res }}$, frequencies $\varepsilon_{i}^{\text {frq }}$, divergence rates $\varepsilon_{i}^{\text {div }}$, and shapes $\varepsilon_{i}^{\mathrm{mac}}$ previously defined are used (which corresponds respectively to the res, frq, div and mac labels on the $\mathrm{x}$ axis of each figure). For the errors on frequencies, divergence rates and shapes, the reference modes are the complex modes presented in section 4 and computed by using a residue iterative algorithm. In the CMS bases, the maximal number of component modes is taken into account (see table 3 at $10 \mathrm{kHz}$ ). In the bases constructed from the real coupled modes, a number of normal modes $n_{R}=66$ is generally used which corresponds to a truncation frequency of $6 \mathrm{kHz}$. A test with a more extended real base with $n_{R}=130$ modes, i.e. a truncation frequency of $10 \mathrm{kHz}$, is also performed for comparison.

As for the frictionless case, the maximum errors for each kind of mode are given but unstable and stable pins modes are distinguished instead of F1 and T1 pin modes. Indeed, the problem of pertinence of the shape criterion $\varepsilon_{i}^{\mathrm{mac}}$ for multiple modes is also encountered here for some sets of stable F1 pin modes which are very close to each other. They are grouped together with the T1 pins modes which are still not affected by the contact, even with friction (cf. table 1). Except this particular problem for some quasi-multiple stable pins modes, the different errors criteria give rather coherent results. However, errors on mode shapes tend to decrease with even more difficulty than in the frictionless case and errors on divergence rates seem to be very sensitive in some cases.

The results obtained when using the CMS or real classical bases are acceptable, but it is clear that adding new static modes adapted to friction improves their performances. This is particularly true for the bases built from free components modes or real coupled modes (cf. figure 14 and 17) which share a very good initial description of the dynamics of the frictionless system. With the newly introduced static modes, all errors are reduced by a factor comprised between 10 and 100 both for the disc modes and the unstable pins modes. On the other hand, the extension 


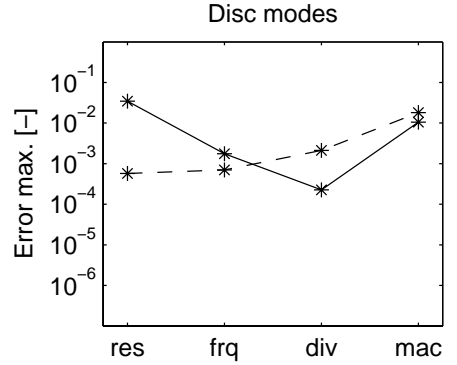

(a)

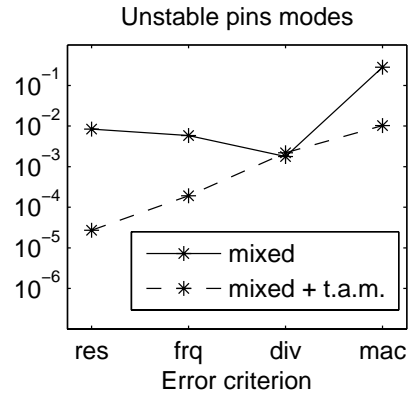

(b)

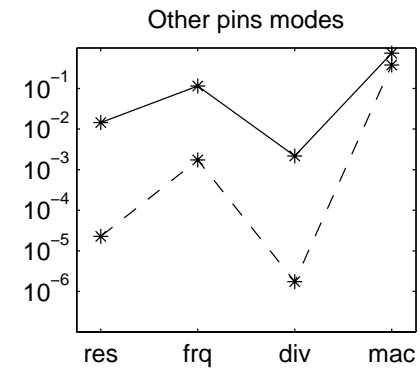

(c)

Figure 16: Performance of the mixed CMS bases for the stability study for: (a) disc modes; (b) unstable pins modes; and, (c) other pins modes

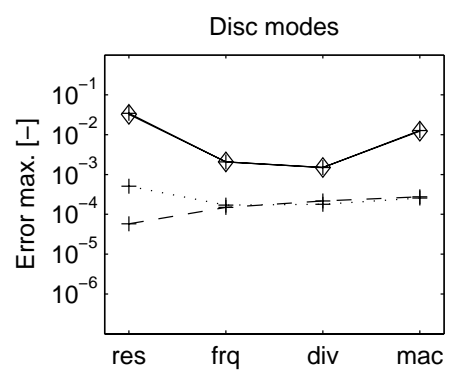

(a)

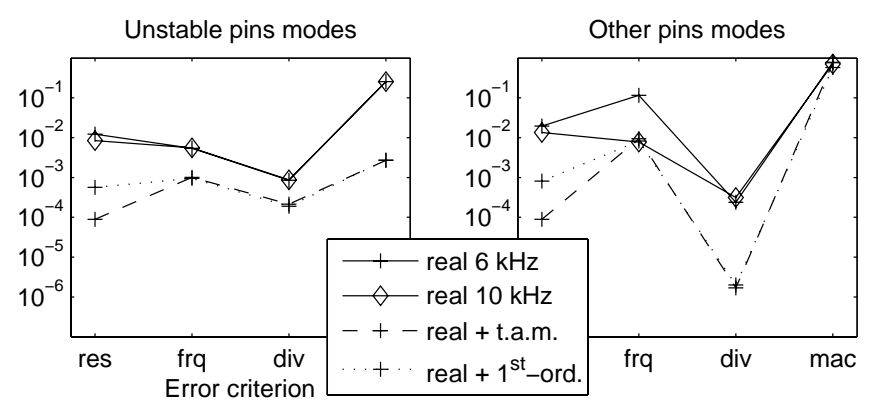

(b)

(c)

Figure 17: Performance of the bases constructed from the real coupled modes for the stability study for: (a) disc modes; (b) unstable pins modes; and, (c) other pins modes 


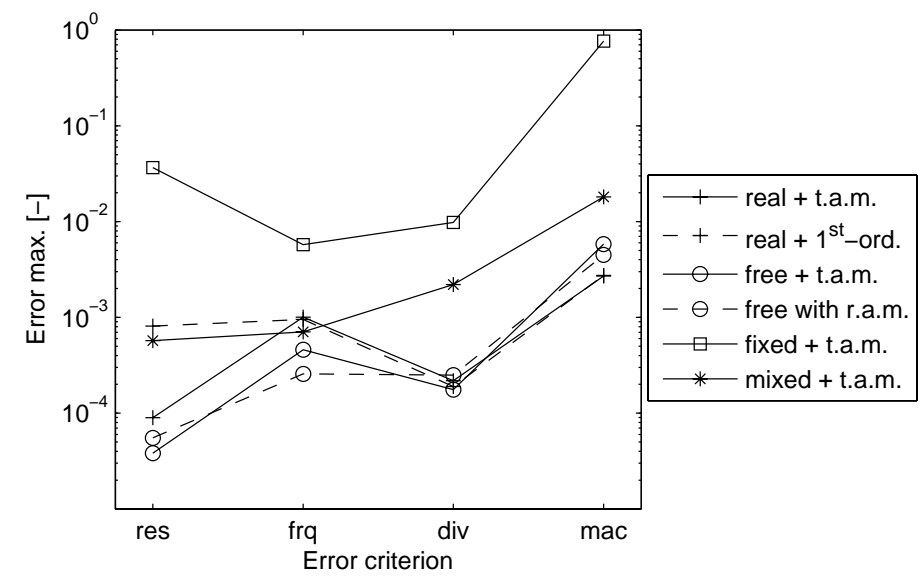

Figure 18: Global comparison of the performances of the enriched bases for the stability study

to $10 \mathrm{kHz}$ of the basis composed of real coupled modes has no significant effect on the errors. The proposed enrichment is thus more adapted than a simple increasing of the number of modes taken into account.

As expected, the fixed CMS basis, which was not efficient for the frictionless problem, still exhibits poor performances for the stability study even if enriched (cf. figure 15). The mixed basis gives intermediate results (cf. figure 16). Without tangential attachments modes, it provides surprisingly low errors for the divergence rates of disc modes. As a consequence, when it is enriched, the errors are reduced except for these divergence rates. However, when considering the maximal error for both disc and pins modes, the enriched basis is at least as effective as the initial one whatever the criterion.

A global comparison of the enriched bases is given on figure 18. For this comparison, the maximum error on both the disc and unstable pins modes is taken into account. It appears that the performances of the bases built from free components modes and real coupled modes are similar. From a practical point of view, these are the only bases which provide a maximal error on divergence rates lower than $0.1 \%\left(10^{-3}\right.$ in absolute value on figure 18) which seems to be necessary to correctly determine the stability of a lightly damped structure. In table 6 , the size $n_{r}$ of these four bases, as well as the total computational time $t_{r}$ (including the building of the base) needed to calculate the complex modes are given. Relative indicators are also calculated:

- the size gain of the basis $G_{n}=\frac{n}{n_{r}}$ where $n=30942$ is the number of degrees of freedom of the whole structure,

- the computational time gain of the basis $G_{t}=\frac{t}{t_{r}}$ where $t=3242 \mathrm{~s}$ is the time needed to solve the complex eigenvalue problem without reduction.

For these four bases, the order of magnitude of the size gain is 100 whereas the cpu time gains are all greater than 20 , which are both considerable values. The use of real coupled modes combined with a $1^{\text {st }}$-order approximation of the response to frictional forces is a very interesting option, providing the highest gains both in size and cpu time. If, for some external constraints like experimental updating or damping modeling, components modes are preferred, the use of rubbing attachments modes seems to be a good technique to complete the free-interface components normal modes. Indeed, in this case, the size of the basis depends only on the number of components normal modes and not on the number of contact nodes (cf. table 4). Conversely, the two methods using attachments modes provide bases with higher sizes. However, their time gains are rather similar and for systems where the number of contact nodes is small compared with the number of modes in the frequency range of interest, the use of tangential attachment modes may be a better option to complete either the components modes or the real coupled modes. 


\begin{tabular}{c|cc|cc} 
Method & $n_{r}$ & $G_{n}$ & $t_{r}[\mathrm{~s}]$ & $G_{t}$ \\
\hline free + t.a.m. & 428 & 72 & 142 & 23 \\
free with r.a.m. & 200 & 155 & 108 & 30 \\
real + t.a.m. & 294 & 105 & 124 & 26 \\
real + 1 1 $^{\text {st } \text {-ord. }}$ & 132 & 234 & 113 & 29
\end{tabular}

Table 6: Size and computational time gains of the four retained bases

\section{Conclusion}

The complex eigenvalue analysis is a widely used technique to investigate the stability of a dynamical system with frictional contact. In the case of brake systems, it is the most frequently employed method to study the propensity of the brake to generate squeal noise. When iterative solvers are used, the complex modes and eigenvalues are calculated with good precision. In practice, reduced real bases are often used in order to save computational times. In this case, great attention should be focused on the choice of the basis. Indeed, it has been shown that classical CMS techniques or bases composed of real coupled modes may lead to significant errors in terms of frequency, divergence rates and shapes. In particular, errors on divergence rates greater than $0.1 \%$ lead to an erroneous prediction of the stability of a lightly damped system which may have important practical consequences.

In this work, some enriched bases are proposed in order to improve the precision on the calculated complex modes and eigenvalues. When bases built from component modes are used, it is suggested to add tangential attachment modes to classical CMS bases or to use the new rubbing attachment modes defined in the paper. In the case of the disc/pads system studied here, such enrichments on free-interface CMS bases allow a significant decrease of the errors on frequencies, divergences rates and shapes by a factor comprised between 10 and 100. If real coupled modes are preferred, the use of tangential attachments modes is also an option but another technique is proposed. It consists in including in the basis the static response to a first-order approximation of the friction forces around the real coupled modes. Applied to the present disc/pads model, the two enrichment options provide errors about 10 times smaller than the initial basis. This is an interesting gain since errors on divergence rates are reduced to about $0.01 \%$. Finally, it must be noted that the computational time gains obtained with these enriched reduced bases remain very high compared with iterative solvers. The corresponding cost/precision ratio is thus optimized.

\section{References}

[1] H. Ouyang, W. Nack, Y. Yuan, F. Chen, Numerical analysis of automotive disc brake squeal: a review, International Journal of Vehicle Noise and Vibration 1 (2005) 207-231.

[2] D. A. Crolla, A. M. Lang, Brake noise and vibration - State of the art, in: Vehicle tribology, number 18 in Tribology series, Leeds, England, pp. $165-174$.

[3] S. Yang, R. F. Gibson, Brake vibration and noise: reviews, comments and proposals, International Journal of Materials and Product Technology 12 (1997) 496-513.

[4] A. Papinniemi, J. C. S. Lai, J. Zhao, L. Loader, Brake squeal: a literature review, Applied Acoustics 63 (2002) 391-400.

[5] N. M. Kinkaid, O. M. O'Reilly, P. Papadopoulos, Automotive disc brake squeal, Journal of Sound and Vibration 267 (2003) $105-166$.

[6] A. Akay, Acoustics of friction, Journal of the Acoustical Society of America 111 (2002) 1252-1548.

[7] R. A. Ibrahim, Friction-induced vibration, chatter, squeal, and chaos part II: Dynamics and modelling, Applied Mechanics Review 47 (1994) 227-253.

[8] N. Hinrichs, M. Oestreich, K. Popp, On the modelling of friction oscillators, Journal of Sound and Vibration 216 (1998) $435-459$.

[9] M. T. Bengisu, A. Akay, Stability of friction-induced vibrations in multi-degree-of-freedom systems, Journal of Sound and Vibration 171 (1994) 557-570.

[10] G. Fritz, J.-J. Sinou, J.-M. Duffal, L. Jézéquel, Investigation of the relationship between damping and mode-coupling patterns in case of brake squeal, Journal of Sound and Vibration 307 (2007) $591-609$.

[11] J. T. Oden, J. A. C. Martins, Models and computational methods for dynamic friction phenomena, Computer Methods in Applied Mechanics and Engineering 52 (1985) 527-634.

[12] B. Hervé, J.-J. Sinou, H. Mahé, L. Jézéquel, Extension of the destabilization paradox to limit cycle amplitudes for a nonlinear self-excited system subject to gyroscopic and circulatory actions, Journal of Sound and Vibration 323 (2009) 944 - 973.

[13] H. Ouyang, J. E. Mottershead, Unstable travelling waves in the friction-induced vibration of discs, Journal of Sound and Vibration 248 (2001) 768-779.

[14] X. Lorang, F. Foy-Margiocchi, Q. Nguyen, P.-E. Gautier, TGV disc brake squeal, Journal of Sound and Vibration 293 (2006) $735-746$.

[15] N. Coudeyras, J.-J. Sinou, S. Nacivet, A new treatment for predicting the self-excited vibrations of nonlinear systems with frictional interfaces: The constrained harmonic balance method, with application to disc brake squeal, Journal of Sound and Vibration 319 (2009) 1175 - 1199. 
[16] D. C. Sorensen, Numerical methods for large eigenvalue problems, Acta Numerica 11 (2002) 519-584.

[17] A. Bobillot, Méthode de réduction pour le recalage. Application au cas d'Ariane 5 (Reduction method for model updating. Implementation on Ariane 5 case), Ph.D. thesis, Ecole Centrale de Paris, 2002.

[18] R. R. J. Craig, M. C. Bampton, Coupling of substructures for dynamic analyses, AIAA Journal 6-7 (1968) 1313-1319.

[19] R. H. Mac Neal, A hybrid method of component mode synthesis, Computer and structures 1 (1971) 581-601.

[20] S. Rubin, Improved component mode synthesis for structural dynamic analysis, AIAA Journal of modal analysis 13 (1975) $995-1006$.

[21] R. R. J. Craig, C. J. Chang, On the use of attachment modes in substructure coupling for dynamic analysis, in: AIAA-ASME 18th Structures, Structural Dynamics and Material Conference - Paper 77-405, San Diego, pp. 89-99.

[22] R. R. J. Craig, A review of time-domain and frequency-domain component-mode synthesis, International Journal of Analytical and Experimental Modal Analysis 2 (1987) 59-72.

[23] F. Moirot, Etude de la stabilité d'un équilibre de Coulomb - Application au crissement des freins à disque (Stability study of a Coulomb equilibrium - Implementation on disc brake squeal), Ph.d. thesis, Ecole Polytechnique, France, 1998.

[24] D. Hochlenert, G. Spelsberg-Korspeter, P. Hagedorn, Friction induced vibrations in moving continua and their application to brake squeal, Journal of Applied Mechanics 74 (2007) 542-549.

[25] C. Morales, Comments on the mac and the nco, and a linear modal correlation coefficient, Journal of Sound and Vibration 282 (2005) $529-$ 537.

[26] O. Chiello, X. Lorang, J.-F. Cordier, F. Margiocchi, Experimental characterization and numerical modelling of the squeal noise generated by railway disc brakes, in: Proceedings of the 19th International Congress on Acoustics (ICA 2007), Madrid, Spain.

[27] O. Chiello, X. Lorang, Numerical investigations into the squeal propensity of a railway disc brake, in: 7th European Conference on Noise Control (Euronoise 2008), Paris, France, pp. 1131-1136.

[28] X. Lorang, O. Chiello, Stability and transient analysis in the modelling of railway disc brake squeal, in: Noise and vibration mitigation for rail transportation systems, Notes on Numerical Fluid Mechanics and Multidisciplinary Design, Springer, 2008 , pp. $447-453$.

[29] R. Lehoucq, D. Sorensen, C. Yang, ARPACK Users' Guide: Solution of Large-Scale Eigenvalue Problems with Implicitly Restarted Arnoldi Methods, SIAM Publications, Philadelphia, 1998.

[30] A.-S. Plouin, E. Balmes, Steel/viscoelastic/steel sandwich shells, computational methods and experimental validations, in: Proceedings of the International Modal Analysis Conference (IMAC), Orlando, Florida, USA, pp. 384-390. 